and swollen lips. Following parenteral administration of epinephrine and glucocorticoid together with intravenous bolus of fluids the boy recovered. Two hours earlier he had eaten tuna steak. His mother tasted a piece of it and reported mild tongue pruritus.

Therefore the boy was diagnosed of having scombroid poisoning. Two days later he performed some experiments at home. He realized that his hand in a container with cold water, as opposed to a hand in warm water, has swollen and blushed. When he drank a small amount of an ice tea he felt throat tightness. Barefoot walking on the cold floor caused to him itching of the soles of the feet. The ice cube test was performed at hospital. The test resulted positive and diagnosis of cold urticaria and anaphylaxis was made.

For the whole next year the boy suffered from hypersensitivity reactions to cold. Continuous oral antihistamine therapy made symptoms less severe.

However, it is interesting that the boy got over an acute enteral infection caused by Campylobacter jejuni just a week before the reported episode of anaphylaxis to cold occured. Moreover, two days before the symptoms of the enteral infection started the boy swam in a pool with very cold water (colder than sea water) and had no reactions. Conclusion: The exact mechanisms of hypersensitivity reaction and histamine release to cold are not completely elucidated. There are many infective agents that have already been associated with cold urticaria but Campylobacter jejuni was not one of them. Future investigations are needed to reveal the pathophysiologic pathways that connect these two conditions.

\section{ATYPICAL FOOD-PROTEIN INDUCED ENTEROCOLITIS SYNDROME}

Davor Bandic*. National Memorial Hospital Vukovar, Vukovar, Croatia

10.1136/archdischild-2021-europaediatrics. 153

Food-protein induced enterocolitis syndrome ( FPIES) is a non-IgE mediated gastrointestinal hypersensitivity, categorised into two major phenotypes: acute and chronic. Acute FPIES manifests as profuse, repetitive vomiting, 1-4 hours after exposure to a triggering food, sometimes with diarrhoea 5-10 hours later, leading to dehydration and lethargy. In chronic FPIES symptoms develop over days or weeks with intermittent emesis, progressive diarrhoea, abdominal distension and failure to thrive. The pathophysiology of FPIES remains obscure, but there is evidence of profound activation of innate cells, eosinophils, and pan-T-cell activation. Most FPIES cases are induced by cow's milk proteins and soy in infants, although other single or multiple foods can be triggers, including solid foods, where there are significant geographical differences. The incidence ranges from 0.015 to $0.5 \%$ There is a significant lack of awareness among professionals about FPIES and significant delay of diagnosis. There are some adult patients, too. Diagnosis is purely clinical, and there are diagnostic criteria for patients presenting with possible PFIES and diagnostic criteria for the interpretation of oral food challenges ( OFC ), as shown here. The most important aspect of management in patients with FPIES is the avoidance of triggering food. The natural course is favourable, and there are no reports of longterm complications in children with FPIES and it is largely a self-limiting, generally benign disorder of childhood.
Patients with FPIES usually have undetectable specific IgE to triggering food, except in rare cases of atypical FPIES. In addition to classic acute FPIES symptoms, children with atypical FPIES can also have symptoms consistent with an acute IgE-mediated hypersensitivity such as urticaria, angioedema, flushing, and wheezing, and are likely to have a more protracted course. Rarely, patients with IgE-mediated food allergies develop non-IgE-mediated food allergies and vice versa. Our patient is a child who had been avoiding cow's milk due to immediate cutaneous reaction on ingestion, with positive specific IgE and skin prick-to-prick test. After ten months of avoidance, and negative new specific IgE, she was challenged for resolution. She tolerated cow's milk during the 2 hour observation period, but then developed delayed profuse vomiting. FPIES was confirmed with another, FPIES designed oral food challenge, and she has been advised to continue using an extensive hydrolysate formula. Due to the known possibility of developing FPIES to other proteins, their cautious introduction is advised. Further follow-up will be taken, considering OFC every 18-24 months.

\section{FOOD ALLERGY WITH IDIOPATHIC ANAPHYLAXIS AND MAST CELL ACTIVATION SYNDROME DIAGNOSTIC CHALLENGE}

Mahesh Katre*, Abhidhamma Kaninde, Harsha Bilolikar. Leicester Royal Infirmary, UK

10.1136/archdischild-2021-europaediatrics. 154

Background Food-related anaphylaxis is common in a paediatric age group. Allergic reactions with multisystemic involvement can potentially point towards mast cell activation syndrome.

Case Presentation 6-year-old child initially presented with allergic reaction after eating strawberries. He developed lip swelling, breathing difficulties, itchy rash. Symptoms resolved with oral antihistamine and GP prescribed adrenaline autoinjector (AAI)with suspected anaphylaxis reaction.

At 8 years of age, he had an allergic reaction to orange fruit shoot with lip and tongue swelling, itchy rash. He responded well to oral antihistamine. Similar episode occurred few days later along with breathing difficulty and he received IM adrenaline in the hospital. He also had provocation test with orange fruit shoot and developed anaphylaxis requiring IM adrenaline, IV chlorphenamine.

Later, severe anaphylaxis reactions occurred in school, but there was no identifiable cause found. At one stage, required AAI once a week over 5 weeks period at school with no trigger. On few occasions there was no itchiness but only lip and facial swelling present. He also had chronic urticarial rash for which was prescribe oral cetirizine and was in control.

Once he developed urticarial rash after contact with latex tablecloth in school, after which plan made in place to avoid contact with latex cloth. At this point of time, he was referred to tertiary care centre for further opinion.

Over the next 2 years anaphylactic episodes resolved. Later, developed neurological symptoms of tremor and feeling of being disoriented. School noticed that, his concentration has been lacking. Eye symptoms with redness, itchiness and discomfort were developed.

Investigations Skin prick test showed positive $6 \mathrm{~mm}$, Peanut$3 \mathrm{~mm}$, Walnut-4mm, Hazelnut-4mm, Almond-3mm, Strawberry$3 \mathrm{~mm}$, House dust mite- $2 \mathrm{~mm}$, Birch pollen- $3 \mathrm{~mm}$. Total serum 